\title{
JUURNAL.RU
}

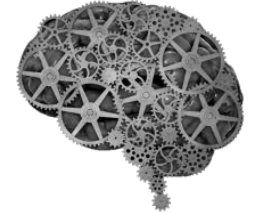

COMPANY GROUP "INTELLEKT"

\author{
Егоров. А.Н., Кузнецов В.А. \\ Государственный университет морского и речного флота \\ имени адмирала С.О. Макарова \\ Санкт-Петербург, Россия
}

doi: 10.18411/lj2016-8-2-05

idsp 000001: lj2016-17-2-05

\section{Высокопроизводительные вычисления на графических процессорах}

\section{Аннотация}

В статье рассматриваются основные преимущества, возможности и тенденции развития технологии использования ресурсов видеокарт для решения задач общего назначения (GPGPU). Особое внимание уделяется программноаппаратной архитектуре CUDA. На основе анализа особенностей обработки данных на графических процессорах, делаются выводы о том, что для достижения максимальной производительности при реализации задач с GPGPU необходимо: оптимизировать работу с памятью устройства, правильно организовать потоки для вычислений и компенсировать ограничения GPU за счёт совместного использования с CPU.

Ключевые слова: GPGPU, параллельные алгоритмы, технология CUDA, высокопроизводительные вычисления.

Во многих направлениях, как в прикладной, так и в научноисследовательских сферах деятельности человека, востребованы высокопроизводительные средства вычислительной техники. Их построение стало возможным не только за счёт улучшения характеристик элементов электронных схем, но и совершенствованию методов обработки больших объёмов данных, в области которой наиболее актуальной и перспективной является распараллеливание вычислительного процесса. Опыт показывает, что организация параллельного выполнения алгоритмов, с целью обеспечении 
максимальной производительности, является важной самостоятельной проблемой, что обуславливает наличие множества подходов для её реализации. Среди прочих, относительно новым, но многообещающим является технология использования ресурсов графических видеокарт (многодетности профессора и оперативной памяти) для вычислений общего назначения GPGPU (англ. Generalpurpose graphics processing units - GPU общего назначения).

Первые поколения видеопроцессоров ориентировались только на работу с графикой, обработка которой реализовывалась исключительно аппаратно, и не имели возможности запуска произвольных алгоритмов. С развитием технологий и увеличением количества методик обработки изображения стало ясно, что аппаратная реализация уступает программной в своей универсальности. Так, например, вычисления нацеленные на получение нестандартные видеоэффекты, приходилось выполнять на центральном процессоре. С появлением шейдеров, программ, предназначенных для исполнения процессорами видеокарты, в третьем поколении и расширением их возможностей в последующих, $\mathrm{y}$ разработчиков появилась возможность реализации собственных алгоритмов обработки графики, что дало начало технологии GPGPU [1].

Выполнение шейдеров на видеокарте осуществляется через графические API (Direct3D и OpenGL) [1, 2]. Используя их для произвольных вычислений необходимо адаптировать исходные данные и алгоритм задачи в вид пригодный для обработки графики, что, несомненно, вызывает трудности при реализации.

Упростить программирование GPGPU позволило появление BrookGPU [1], который по-прежнему использует графические API для взаимодействии с устройством, но скрывает их составляющие при программировании, что даёт возможность реализации алгоритмов на GPU применяя привычные, для вычислений общего назначения, структуры данных и методы их обработки. Появление BrookGPU дало толчок к популяризации GPGPU.

Несомненным плюсом Direct3D и OpenGL является их поддержка видеокартами различных производителей. Однако они имеют ограничения объёма массивов данных и набора инструкций при взаимодействии с устройством [2]. Решением этих проблем стал выпуск низкоуровневых платформ GPGPU: CUDA (Compute Unified Device Architecture) компанией NVIDIA и AMD FireStream (ранее Close-To-Metal) от AMD. Теперь работа с 
аппаратной частью осуществляется непосредственно через драйвер, минуя графические API [1], что даёт широкий доступ к её возможностям, например, позволяет реализовать взаимодействие между параллельными потоками, что ранее было не возможно.

В свою очередь Microsoft выпустил новый API DirectCompute, предназначенный непосредственно для вычислений общего назначения на GPU, входящий в состав DirectX. Вместе с тем, группа компаний Khronos разработала стандарт OpenCL - целью которого обобщить параллельные вычисления на различных устройствах [3]. Сегодня существует поддержка CPU, GPU, а так же карт FPGA. По сравнению с графическими API, DirectCompute и OpenCL предоставляют возможности более гибкого программирования видеокарт, однако, в силу своей универсальности менее эффективны относительно низкоуровневых платформ, т.к. не используют всех особенностей каждого типа устройств. Так по результатам тестирований представленных в [4] реализация на основе OpenCL проигрывает в производительности реализации CUDA на 13$65 \%$, в зависимости от алгоритма.

Рассмотрим особенности работы видеоадаптеров при решении произвольных задач на примере видеокарт NVIDIA и возможности предоставленные разработчику в платформе CUDA для реализации GPGPU.

В отличии от CPU, применяемых для вычислений общего характера, узконаправленность развития видеокарт сформировало особую архитектуру, ориентированную на максимально эффективную обработку графики. Подобные задачи характеризуются выполнением однотипных действий на множественном объёме данных, поэтому GPU реализует SIMD архитектуру $[1,5]$, нацеленную на использование наибольшего количества параллельных потоков, до нескольких тысяч, для расчётов.

Одна из основ технологии CUDA [2] - использование GPU в качестве сопроцессора CPU. В силу различий архитектуры, процессоры отличаются производительностью при решении определённого спектра задач [5]. Например, CPU намного эффективнее выполняет расчёты связанные с множественными переходами и ветвлениями. В свою очередь, GPU отлично справляется с массовым параллелизмом, обрабатывая большие объёмы информации, и не должен применяться к последовательным алгоритмам. Поэтому, для 
компенсации узких мест каждого из устройств, необходимо их совместное использование.

Решение задач на графическом процессоре осуществляется с помощью вычислительного ядра (kernel). CPU должен инициализировать его запуск и задать конфигурацию видеокарты для вычислений, что определяет, в том числе, организацию потоков, совокупность которых делится на блоки (block), объединённые в единую сетку (grid) (рис. 1). Kernel, в свою очередь, содержит процедуры, выполняемые на каждом из множества потоков одной сетки [6]. Следует заметить, что сетка и блоки могут иметь одномерную, двухмерной и трёхмерную структуру [2].

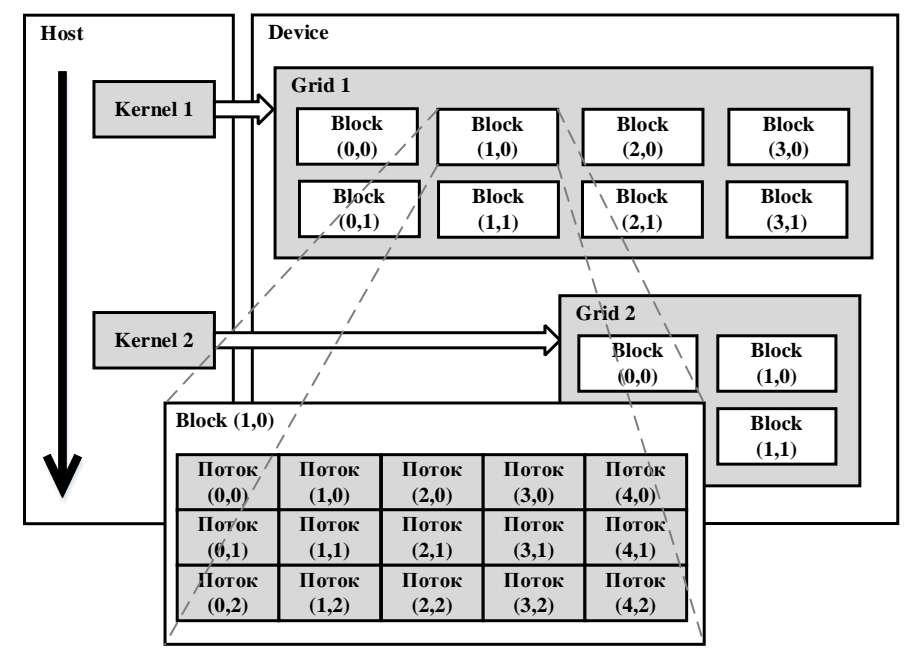

Рис. 1. Вызов kernel, структура сетки и блоков.

Каждый видеоадаптер обладает встроенной оперативной памятью размером достаточным для обработки больших объёмов информации, пропускная способность которой, на много выше ОЗУ используемой CPU [5]. Основное различие заключается в её многоуровневой системе. Существует 6 типов памяти отличных по объёму, скорости чтения/записи и уровню доступа [6]. Правильно организованная работа с памятью один из наиболее важных условий для достижении максимальной производительности вычислений на GPU.

Перед запуском kernel CPU должен скопировать необходимую информацию в память устройства. Для этого host может задействовать: глобальную, константную и текстурную памяти (рис. 2). Глобальная является основной на device, обладает большим объёмом, но высокой латентностью [5], поэтому количество обращений к ней необходимо максимально уменьшить. 
Другие два типа доступны GPU только для чтения и отличаются наличием кэша, что позволяет значительно повысить их эффективность. Текстурная представляет собой особо выделенные участки глобальной памяти [6], чтение которых выполняется методами, ориентированными исключительно на ускорение работы с текстурными данными, что накладывание некоторые трудности при её использовании для вычислений общего назначения. Взаимодействие с константной памятью также тривиально, как и с глобальной, но из-за сравнительного малого размера, её применение ограничено.

GPU дополнительно доступны (рис 2): разделяемая память, объём которой, распределяется между блоками, а так же регистровая и локальная, выделяемые на каждый поток в отдельности. Для всех внутренних переменных, объявленных в kernel, по умолчанию используется регистровая память, обладающая самой высокой скоростью доступа, но малым объёмом. В случае её нехватки задействуется локальная память, сравнимая по эффективности работы с глобальной, чего следует избегать.

Разделяемая память имеет характеристики сравнимые с регистровой. Основным её назначением является обеспечение взаимодействия между потоками внутри блока. Для их синхронизации, например при организации бесконфликтного обращения к разделяемой памяти, предусмотрена функция _syncthreads(), приостанавливающая обработку каждого потока в ожидании остальных.

По завершению вычислений kernel результаты необходимо скопировать из памяти видеокарты и очистить её.

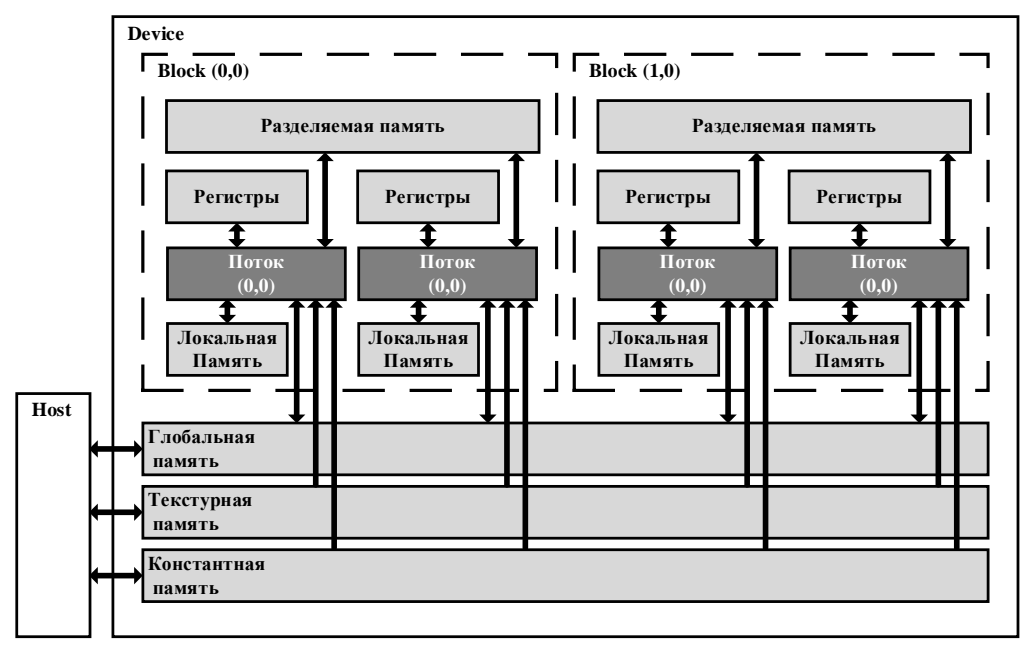

Рис.2 Доступ к различным типам памяти в архитектуре CUDA. 
В силу множества особенностей и ограничений различных типов памяти, оптимизация работы с ними предполагает комплексный подход. Для снижение количества обращений к глобальной памяти существует возможность, при взаимодействии с ней объединить нескольких запросов в единую транзакцию. С той же целью, можно использовать разделяемую память в качестве программируемого кэша, что, помимо прочего, позволит уменьшить нагрузку на регистровую, во избежание использования локальной. Ещё один из возможных вариантов предполагает выполнение декомпозиции алгоритма, реализованного в kernel, на подзадачи, требующие меньшего объёма памяти при вычислениях. И наконец за счёт уменьшение количества потоков в блоке и (или) блоков в сетке, увеличится размер доступной для них памяти, однако, снизится прирост производительности от параллельной обработки данных. Поэтому для принятия конструктивных решений необходимо проанализировать узкие места реализованного алгоритма.

Несомненно, производительность видеоадаптеров высока, но из-за специфики архитектуры, несколько, ограничена. Наиболее важными факторами для достижения максимальной эффективности при реализации задач c применением технологии GPGPU являются: оптимизация работы с памятью устройства, правильная организация потоков и компенсация узких мест GPU за счёт совместного использования с CPU.

Появления возможности использования ресурсов видеокарт для неграфических вычислений позволило не только крупным компаниям, разработчикам, а также специалистам занимающимся научными исследованиями существенно ускорить обработку больших объёмов данных, что уже нашло своё применения в сферах [7]: вычислительной гидродинамики, экономических и финансовых вычисления, анализе данных, обороне и разведке, автоматизации проектирования электронных устройств (EDA), компьютерной оптики, вычислительной химии и биологии, физике и др. 


\section{Литература:}

1. Головашкин Д.Л., Казанский Н.Л. Решение задач компьютерной оптики на графических вычислительных устройствах // Вестник Самарского государственного аэрокосмического университета - Самара: СГАУ - 2010 - №4(24) - C. 159-168.

2. Круглов В.Н., Папуловская Н.В., Чирышев А.В. Преимущества совместного использования CPU и CUDA-устройства // Фундаментальные исследования. - Пенза: издательский дом «Академия Естествознания» 2014. - № 8 (2) - С. 296-304.

3. Баранов Д.А., Влацкая И.В. Анализ эффективности параллельной реализации метода гаусса // Вестник Нижегородского университета им. Н.И. Лобачевского - Нижний Новгород: Нижегородского университета им. Н.И. Лобачевского - 2013, № 1(3) - С. 166-172.

4. Fang J,, Varbanescu A., Sips H.. A Comprehensive Performance Comparison of CUDA and OpenCL // International Conference on Parallel Processing of 13-16 Sept. 2011 - Taipei, Taiwan: P. 216-225.

5. Климов М.И., Меньшов И.С. Эффективный параллельный расчет задач механики сплошной среды для систем гибридной архитектур // Научный Вестник МГТУ ГА - М. МГТУ ГА - 2012 - № 194. - С. 31-39.

6. А. М. Казённов. Основы технологии CUDA // Компьютерные исследования и моделирование - Ижевск: Институт компьютерных исследований (ИКИ) - 2010 - Т. 2, № 3 - С. 295-308.

7. Кузнецов В.А. распараллеливание вычислений для авторматизированной диспетчеризации судопропуска шлюзованной системы // Материалы VII межвузовской научно-практической конференции аспирантов, студентов и курсантов «Современные тенденции и перспективы развития водного транспорта России» 18 мая 2016 года. - СПб.: Изд-во ГУМРФ им. адм. С.О. Макарова, 2016. - С. 263-265. 